\title{
RELEVANCIA DE LOS INVERSIONISTAS INSTITUCIONALES EN EL MERCADO ACCIONARIO COLOMBIANO*
}

\author{
DOI: 10.22395/seec.v20n44a3 \\ Anderson Pino** \\ Jorge M. Uribe ${ }^{* * *}$ \\ Diana M. Jiménez ${ }^{* * * *}$
}

Recibido: 08 de agosto de 2016 • Aprobado: 31 de julio de 2017

\section{RESUMEN}

El objetivo en este artículo es estudiar e identificar la relevancia de los inversionistas institucionales en el mercado de acciones colombiano, usando, para ello, la metodología de las redes, la teoría de los grafos y las matrices de propiedad con información de 108 empresas en el año 2012. Los resultados evidencian un alto grado de concentración de los inversionistas en el mercado accionario; en particular, los fondos de pensiones juegan un papel predominante en el mercado de valores colombiano. Lo anterior es especialmente relevante para un mercado accionario pequeño y poco líquido como el de Colombia, en el cual los inversionistas institucionales juegan como proveedores de liquidez o como instituciones de impacto sistémico, durante períodos de estrés financiero.

\section{PALABRAS CLAVE}

Mercados financieros; inversionistas institucionales; fondos de pensiones; mercados emergentes; riesgo sistémico; Colombia.

\section{CLASIFICACIÓN JEL}

G10, G23, G32

\section{CONTENIDO}

Introducción; 1. Revisión de aspectos teóricos: inversionistas institucionales y su papel en el mercado accionario; 2. Metodología: matrices de participación accionaria y estructura de redes; 3. Principales resultados; 4. Conclusiones; Bibliografía; Anexo.

El presente artículo es el resultado de discusiones durante ejercicios de trabajo entre los grupos de investigación a los que pertenecen los autores.

* Economista, Universidad del Valle, Cali, Colombia. Profesional de Apoyo, Proyecto Municipios en Zonas de Conflicto Fortalecidos en su Institucionalidad para la Paz, Fundación para el Desarrollo y el Progreso. Correo electrónico: anderson. pino@correounivalle.edu.co

... Economista, Universidad del Valle, Cali, Colombia. Profesional en Finanzas y Negocios Internacionales, Universidad Santiago de Cali, Cali, Colombia. Magíster en Economía, Universidad de los Andes, Bogotá, Colombia. Magíster en Economía, European University Institute, Florencia, Italia. Estudiante de Doctorado, Universidad de Barcelona, Barcelona, España. Profesor e investigador y miembro del Grupo de Investigación Macroeconomía Aplicada y Economía Financiera (clasificado por Colciencias en categoría C en 2017), Departamento de Economía, Facultad de Ciencias Sociales y Económicas, Universidad del Valle, Cali, Colombia. Dirección: Calle 13 \# 100-00 Ciudadela Universitaria Meléndez, oficina 3036. Correo electrónico: jorge.uribe@correounivalle.edu.co

.... Economista, Universidad del Valle, Cali, Colombia. Magíster en Economía Aplicada, Universidad del Valle, Cali, Colombia Profesora, investigadora y miembro del Grupo de Investigación Desarrollo Económico, Crecimiento y Mercado Laboral (clasificado por Colciencias en categoría A en 2017), Departamento de Economía, Facultad de Ciencias Sociales y Económicas, Universidad del Valle, Cali, Colombia. Dirección: Calle 13 \# 100 00 Ciudadela Universitaria Meléndez, oficina 3036. Correo electrónico: diana.marcela.jimenez@correounivalle.edu.co 


\section{RELEVANCE OF INSTITUTIONAL INVESTORS IN THE COLOMBIAN EXCHANGE MARKET}

\section{ABSTRACT}

The objective of this article is to study and identify the relevance of institutional investors in the Colombian stock market by using the network methodology, the graph theory on property with information of 108 companies in year 2012. The results show a high degree of concentration of investors on the stock market; specifically, the pension funds play a significant role in the Colombian Stock Market. This is especially relevant for a small and not very liquid stock market as that in Colombia, where institutional investors play like liquidity provides or like systemic impact institutions during financial stress periods.

\section{KEY WORDS}

Financial markets; institutional investors; pension funds; emerging markets; systemic risk; Colombia.

\section{JEL CLASSIFICATION}

G10, G23, G32

\section{CONTENT}

Introduction; 1. Revision of theoretical aspects: institutional investors and their role in the stock market; 2. Methodology: Stock participation matrix and network structure;

3. Main results. 4. Conclusions; Bibliography; Annex.

\section{RELEVÂNCIA DOS INVESTIDORES INSTITUCIONAIS NO MERCADO ACIONISTA COLOMBIANO}

\section{RESUMO}

O objetivo deste artigo é estudar e identificar a relevância dos investidores institucionais no mercado acionista colombiano. Para isso, utilizou-se a metodologia das redes, a teoria dos grafos e as matrizes de propriedade com informação de 108 empresas no ano 2012. Os resultados demonstram alto grau de concentração dos investidores no mercado de ações; em particular, os fundos de pensão desempenham um papel predominante no mercado de valores colombiano. Isso é especialmente relevante para um mercado acionista pequeno e pouco líquido como o da Colômbia, no qual os investidores institucionais atuam como os que fornecem liquidez ou como instituições de impacto sistêmico, durante períodos de estresse financeiro.

\section{PALAVRAS-CHAVE}

Mercados financeiros; investidores institucionais; fundos de pensão; mercados emergentes; risco sistêmico; Colômbia.

\section{CLASSIFICAÇÃO JEL}

G10, G23, G32

\section{CONTEÚDO}

Introdução; 1. Revisão de aspectos teóricos: investimentos institucionais e seu papel no mercado acionista; 2. Metodologia: matrizes de participação acionista e estrutura de redes; 3. Principais resultados; 4. Conclusões; Bibliografia; Anexo. 


\section{INTRODUCCIÓN}

El papel de los inversionistas institucionales (fondos de pensiones, de cobertura de riesgo, fondos mutuos de inversión, bancos comerciales y compañías de seguros) ha sido objeto de intenso estudio en la literatura financiera y económica. Hoy se sabe que estos inversionistas están relacionados con la explicación de diferentes hechos estilizados de las series de tiempo financieras, relacionados con los precios, retornos, volúmenes de negociación y capitalización bursátil, en diferentes mercados y en horizontes de tiempo heterogéneos.

Para el caso colombiano se ha identificado que los inversionistas institucionales están relacionados con importantes dinámicas del mercado, tanto desde el lado de la oferta como desde el lado de la demanda. Por una parte, estos inversionistas parecen estar asociados con el buen desempeño relativo del mercado de acciones en épocas recientes, en términos de capitalización de mercado y liquidez, según el estudio de Uribe, Mosquera y Restrepo (2013). También se han identificado importantes fuentes de vulnerabilidad del mercado de acciones colombiano, en relación con la aparición de choques extremos, en mayor grado, debido a la presencia de conglomerados económicos que capturan porciones significativas de las operaciones del mercado, siguiendo a Uribe y Fernández (2014).

A pesar de lo anterior, la medición de la relevancia de los inversionistas institucionales, dentro del mercado de acciones colombiano, ha sido un punto ausente de la agenda académica nacional. Este documento busca llenar este faltante, mediante la construcción de un modelo empírico de redes sociales. Los objetivos de este estudio apuntan a clarificar el grado de centralidad de los inversionistas institucionales en el mercado colombiano, así como a identificar mecanismos ocultos que generen vulnerabilidad para el sistema, los cuales podrían aparecer después de enfrentar la quiebra de una o más entidades centrales en el mismo.

Existen importantes precedentes en la literatura económica, en autores como Gompers y Metrick (2001), Cohen, Gompers y Vuolteenaho (2002), Dennis y Strickland (2002), Hvidkjaer (2006) o Brunnermeier y Nagel (2004), que vinculan a los inversionistas institucionales con la determinación del precio de los activos financieros; otros más, con problemas de suministro de liquidez y con el riesgo de mercado, como es el caso de Gabaix et al. (2006).

Este estudio emplea un análisis de la topología de la red que describe el mercado de acciones en Colombia. El análisis propuesto se basa en la construcción de matrices de participación accionaria cruzada y redes, así como de estadísticos de centralidad y conectividad de la red. También involucra análisis de simulación 
que toman la red empírica construida como punto de partida. Esta metodología permite identificar mecanismos de transmisión en el sistema, que de otra forma podrían pasar desapercibidos.

Los resultados del estudio, tanto los referentes a la caracterización de la red y la identificación de las entidades con mayores nodos de entrada y salida del sistema, como los ejercicios de simulación desarrollados, resaltan la importancia de los fondos de pensiones como agentes de evidente importancia sistémica para el mercado accionario colombiano. Una eventual falla en la operación de alguna de estas entidades implicaría un choque para el mercado de elevada magnitud, contra el cual sería virtualmente imposible cubrirse en el mercado local, lo cual pondría en juego la estabilidad del sistema como un todo.

Este este documento se organiza así: en la segunda parte se presenta un breve recuento teórico del problema de estudio. En la tercera se expone la metodología utilizada; en la cuarta se presentan los principales resultados. Las conclusiones, junto con algunas recomendaciones de política y posibles líneas de investigación futura se presentan en la última sección.

\section{REVISIÓN DE ASPECTOS TEÓRICOS: INVERSIONISTAS INSTITUCIONALES Y SU PAPEL EN EL MERCADO ACCIONARIO}

Como bien lo hacen Gompers y Metrick (2001), ya está documentado que los inversionistas institucionales tienen especial relevancia al explicar la dinámica de baja frecuencia de los precios de los activos financieros, en particular de las acciones. De paso, autores como Lakonishok, Shleifer y Vishny (1992), Choe, Kho y Stulz, (1999), Cohen, Gompers y Vuolteenaho (2002), Dennis y Strickland (2002) y Hvidkjaer (2006), también relacionan a este tipo de inversionistas, con las explicaciones de los fenómenos de momentum que se han encontrado en distintos mercados financieros, debido a las estrategias de feedback trading que suelen emplear y que consisten en tomar posiciones largas, en los activos con rentabilidades positivas en el período anterior, y posiciones cortas, en los activos que registraron rentabilidad negativa. De otro lado, Brunnermeier y Nagel (2004) también aportan evidencia sobre su relación con la aparición de burbujas especulativas en los precios de los activos, así como con los colapsos que suelen sucederlas; Campbell, Ramadorai y Schwartz, (2009) exponen sobre problemas derivados de consideraciones relacionadas con la provisión de liquidez en el mercado y con incrementos en los niveles de volatilidad y de correlaciones cruzadas entre activos pertenecientes a índices de mercado, desde una perspectiva empírica, como lo hacen Harris y Gurel (1986), Shleifer (1986), Chen, Noronha y Singal (2004), o como Basak y Pavlova (2013) que lo abordan desde lo teórico. 
Para autores como Sias (1996), Dennis y Strickland (2002), Gabaix et al. (2006) también los inversionistas institucionales parecen responsables, en gran medida, de la explicación de volatilidades excesivamente altas en los precios de las acciones, así como de picos en los precios y volúmenes de transacción en el mercado. No obstante, la evidencia al respecto no es conclusiva ya que algunos estudios como los de Bohl, Brzeszczynski y Wilfling (2009) y Thomas, Spartaro y Mathew (2014) encuentran que, en algunos escenarios, los inversionistas institucionales pueden actuar como moderadores de la volatilidad del mercado, contribuyendo con estrategias de inversión más informadas que las de los inversionistas no institucionales.

Por otra parte, los inversionistas institucionales juegan un papel central en la configuración de los mercados de acciones menos desarrollados. Según Niggemann y Rocholl (2010) y Chan-Lau (2005) existe evidencia de que en este caso los fondos de pensiones impulsan el crecimiento del mercado, contribuyendo con un incremento sostenido de la liquidez mientras Schmidt-Hebbel (1999), Davis y Hu (2005) exponen que en algunas ocasiones contribuyen, pero con un incremento del ahorro nacional.

En el caso colombiano, Uribe, Mosquera y Restrepo (2013) han identificado que los inversionistas institucionales están relacionados con el buen desempeño relativo del mercado de acciones en términos de capitalización de mercado y liquidez. A su vez, Uribe y Fernández (2014) muestran que estos inversionistas son importantes fuentes de vulnerabilidad del mercado accionario, relacionándolos con la aparición de choques extremos, en mayor grado, debido a la presencia de conglomerados económicos que dentro de su operación cuentan con administradoras de valores y fondos de pensión que capturan porciones significativas de las operaciones del mercado.

A pesar de lo anterior y que según los balances registrados en la página de la Superintendencia Financiera de Colombia', los activos de los cinco fondos de pensiones más grandes en Colombia han presentado un incremento significativo en años recientes, en términos del PIB nacional, y han pasado de aproximadamente un $50 \%$ en 2006, a más del $90 \%$ en 2014, la academia nacional poca atención ha prestado a la medición de la centralidad de los inversionistas institucionales.

Así, en este documento se intenta medir tal centralidad mediante la construcción de un modelo empírico teniendo en cuenta la teoría de las redes sociales y la teoría de los grafos. A su vez, el modelo está basado en matrices de propiedad accionaria, directa e indirecta, lo que permite analizar la topología de la red, es decir, analizar

1 En la página web de la Superintendencia Financiera de Colombia se puede consultar la información financiera sobre las administradoras de fondos de pensiones y cesantías, así como la información sobre los respectivos fondos que administran. 
los grados de completitud e interconectividad de la red para luego, mediante simulaciones de desaparición de nodos, dimensionar la importancia de los inversionistas institucionales (fondos de pensiones, bancos comerciales) dentro de la estructura de la red. Gracias a esta metodología, también se identifican mecanismos ocultos de vulnerabilidad en el sistema, que podrían aparecer después de enfrentar la quiebra de una o más entidades centrales para el mismo.

El presente documento, además de estar relacionado con los planteamientos dados sobre el papel de los inversionistas institucionales en el mercado de títulos valores, también puede ser visto como un paso más en la búsqueda de una metodología adecuada para la identificación de instituciones de importancia sistémica. Tales instituciones requieren, por su naturaleza, consideraciones especiales en términos de regulación y solvencia, en momentos de estrés financiero, debido a las consecuencias que su mal funcionamiento pudiera acarrear sobre el mercado como un todo. En este aspecto la literatura internacional ha avanzado ampliamente en épocas recientes Iver por ejemplo los estudios de Acharya et al. (2010) y Adrian y Brunnermeier (2014)], también con algunos avances en el ámbito nacional, mayoritariamente de investigadores del Banco de la República (ver Cabrera, Melo y Parra (2014), Cabrera, Corredor y Quicazan (2012), Machado et al. (2011) y Cepeda (2008).

Por otro lado, desde el enfoque del análisis de redes, León y Pérez (2013) evalúan la importancia de la infraestructura del sistema financiero para el mercado colombiano, a partir de la estimación de dos medidas de centralidad: authority centrality y hub centrality, cuyos resultados permiten identificar la intensidad de la supervisión y regulación del mercado. Pérez, León y Mariño (2015) realizan una caracterización del mercado cambiario a partir de las principales medidas de centralidad: centralidad de grado, centralidad de grado ponderada, centralidad de eigenvector y centralidad de autoridad y distribución, y encuentran que el mercado cambiario puede ser catalogado como una red de estructura jerárquica donde un grupo pequeño (amplio) de intermediarios posee una amplia (reducida) proporción del número de transacciones. Por último, León, Machado y Sarmiento (2014) modelan la asignación de liquidez del Banco Central colombiano entre los participantes del mercado interbancario, y encuentran que el mercado de fondos interbancarios presenta una estructura de red no homogénea y jerárquica, y confirman que la probabilidad de ser un súper-spreaders está condicionada por el tamaño de las instituciones financieras.

\section{METODOLOGÍA: MATRICES DE PARTICIPACIÓN ACCIONARIA Y ESTRUCTURA DE REDES}

En la primera parte de la metodología empleada se explica cómo construir una matriz de participaciones directas e indirectas, que modele de forma completa la estructura de control cruzada dentro de las firmas que pertenecen al mercado de 
acciones colombiano (o incluso algunas entidades por fuera de este). En la segunda parte se construye una red estática con la información que proviene del primer punto. La construcción de la red estática se prefiere en este caso sobre una alternativa dinámica, de mayor complejidad, puesto que las matrices de participación, tal y como se explica más adelante, ya recogen, por definición, la dinámica del sistema. No obstante, algunos ejercicios futuros podrían incluir la construcción de redes dinámicas, con el fin de analizar propuestas que quedan por fuera del alcance del presente estudio, sobre las que se discutirá en las conclusiones.

\subsection{Matrices de participación}

El estudio sobre el control o la propiedad última de una firma empieza con la pregunta fundamental sobre quién posee realmente una firma: el inversionista que mantiene la mayoría de las participaciones directas en esta o el inversionista que la controla a través de las cadenas de participaciones y derechos a votos indirectos. Siguiendo a Grossman y Hart (1986) se ha identificado a los propietarios de la firma como aquellos que la controlan directa o indirectamente, alineándose entonces con la segunda opción.

Las matrices de control que emergen de esta discusión sirven para caracterizar la estructura de participaciones cruzadas directas e indirectas en el mercado colombiano. Para explicar de forma intuitiva en qué consisten tales matrices de propiedad accionaria, considérese el caso de un accionista que tiene participación en dos empresas A y Z. Supóngase que este inversionista posee el 50\% de cada empresa. Desde el punto de vista de la participación directa (también conocida como la perspectiva del flujo de caja), este accionista recibirá el 50\% de las ganancias de cada empresa. Si en un escenario hipotético la empresa A incrementara sus beneficios en $\mathrm{X}$ pesos, los beneficios del accionista se incrementarían, debido a su control directo sobre tal firma, en exactamente $0.5^{*} \mathrm{X}$ pesos.

No obstante, el punto de vista del flujo de caja puede resultar incompleto a la hora de caracterizar la estructura de propiedad real en un mercado. Es así como existe una corriente de la literatura que busca caracterizar el control accionario último (por medio de votos) y para la cual no solo es relevante la participación directa del accionista sino que será necesario incluir el control indirecto que el mismo ejerce sobre la empresa B, dada su participación en la misma a través de la empresa A. Es decir, el accionista controla el 50\% de B de forma directa, y al menos el 25\% de forma indirecta (ver gráfico 1). Por supuesto, el análisis se podría llevar un paso más lejos para incluir la participación que aparecería después de realizar una iteración más, para tener en consideración el control que el accionista ejerce en la empresa 
A, a través de su participación en la empresa B, y así sucesivamente. Es en esta segunda corriente de la literatura donde se apoya la primera parte de la metodología de este estudio. Para una revisión más detallada del tema se recomienda el estudio de Glattfelder (2013) y una aplicación para el caso colombiano en Gutiérrez, Pombo y Taborda (2008).

\section{Gráfico 1. Esquema de participación directa e indirecta}
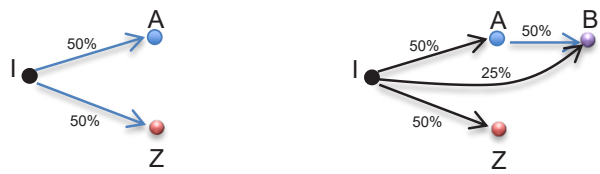

Fuente: elaboración propia

La metodología aquí expuesta se basa en el hecho de que la iteración, a través de multiplicaciones sucesivas, de las matrices de participación accionaria directa, por fuerza, converge a una matriz límite. Esta matriz límite estará compuesta por entradas que representan el control accionario último de cada empresa sobre las demás, después de tener en cuenta toda la cadena de iteraciones de la propiedad.

Formalmente, el punto inicial en la metodología es la definición de una matriz A de tamaño $(\mathrm{N} \times \mathrm{N})$ :

$$
\mathrm{A}=\left[a_{i j}\right]
$$

Siendo $a_{i j}$ cada elemento de la matriz A, donde se registra la participación de la empresa $i$ en el balance de la empresa $j$. Como se habla de participaciones, los elementos de esta matriz cumplen, por construcción, con las siguientes tres propiedades:

i. $0<a_{i j}<1$

ii. $a_{i j}=0$ si $i=j$, donde $i=1, \ldots, \mathrm{N} ; \mathrm{y} j=1, \ldots$; y finalmente,

iii. $\sum_{i=1}^{\mathrm{N}} a_{i j} \leq 1$

La primera y la última propiedades corresponden a los casos de alguna participación accionaria de la empresa $i$ en $j$, y mayoritaria participación accionaria, respectivamente. 
Ahora, la propiedad integrada en el modelo se define como la suma de la propiedad directa y la indirecta. La matriz con las participaciones total es:

$$
\mathrm{Z}=\left[z_{i j}\right]
$$

Que estará dada por la solución al sistema:

$$
\mathrm{Z}=\mathrm{A}+\mathrm{AZ}
$$

Que dada la propiedad $i$, antes mencionada, es igual a

$$
\mathrm{Z}=\mathrm{A}(\mathrm{I}-\mathrm{A})^{-1} \text {, }
$$

Donde I es la matriz identidad de orden $(\mathrm{N} \times \mathrm{N})$. La ecuación [7] es útil para computar la propiedad integrada de grupos económicos jerárquicos y sirve como insumo para construir una red que permita identificar instituciones importantes en términos de riesgo sistémico.

\subsection{Enfoque de redes}

Una red $\mathrm{G}$ es una estructura conformada por un conjunto de nodos, $\mathrm{N}$, en el que se encuentran los agentes objeto de estudio que, en este caso, son las empresas que emiten y compran acciones en el mercado de valores colombiano, y un conjunto $\mathrm{E}$, con los enlaces que se conforman entre los nodos, lo que para este análisis representa las participaciones cruzadas de las empresas o la propiedad integrada tal y como se define en la matriz de la expresión [7].

Gracias a la metodología de las redes y su correspondiente complemento matemático la teoría de los grafos se puede, dada la estructura emergente de red, resaltar la importancia del control accionario último de cada empresa en otras y, además, detectar las firmas más relevantes en la red a través del cálculo de medidas de centralidad, poder y prestigio. La medida de centralidad está apoyada en el concepto de grado del nodo y este último representa el número de enlaces que una empresa tiene con otras:

$$
d\left(n_{i}\right)=\sum_{j=1}^{E} a_{i j} \text {, para una red no dirigida. }
$$

Sin embargo, el grado de nodo, como medida de grafos no dirigidos, ofrece poca información en este análisis, pues no permite discriminar cuántas y cuáles son las empresas en las que una firma específica tiene propiedad integrada, ni las empresas que tienen control accionario último en dicha firma específica. Para superar lo anterior, y bajo la versión dirigida de la red emergente, para el conjunto de firmas 
analizadas, de $d\left(n_{i}\right)$ se puede obtener el grado de entrada o grado de salida de un nodo. El primero es el número de enlaces incidentes -que llegan-al nodo mientras el segundo es el número de vínculos que salen de él. El grado nodal de entrada [9] da información del número de firmas con control accionario último en la empresa $i$ mientras que el grado de salida [10] determina la cantidad de firmas en la que $i$ tiene propiedad integrada.

$$
\begin{aligned}
& d\left(n_{+, i}\right)=\sum_{+=1}^{E} a_{+, i} \text { para }+\neq i, \\
& d\left(n_{i,+}\right)=\sum_{+=1}^{E} a_{i,+} \text { para }+\neq i .
\end{aligned}
$$

La representación gráfica de los grados de entrada y de salida se hace a través de las redes de ego de cada firma; en ellas se detallan cuáles son las empresas en las que se tiene control accionario último (grado de salida) y cuáles son las firmas que tienen propiedad integrada en cada empresa (grado de entrada).

Otra medida de centralidad y prestigio es la de autoridad. Como bien lo plantea Kleinberg (1999) en las estructuras de red se pueden encontrar dos tipos de nodos centrales: el primer tipo de nodo es el de distribución (Hub), y está relacionado con los nodos más importantes en la red. El segundo tipo de nodos son de autoridad (Authority) donde se contactan los nodos más importantes de la red; o, en otras palabras, dado que la importancia de un nodo está definida por la cantidad de enlaces que tenga con nodos cuyos grados nodales sean altos, si un nodo es una autoridad, es porque a él lo conectan nodos que tiene muchas conexiones (ver gráfico 2).

Para este análisis, las empresas que son autoridad son aquellas que presentan los grados más altos de entrada; por lo tanto, son las firmas cuyo control accionario último está concentrado en las empresas que tienen conexión hacia ella. Adicionalmente, la medida de autoridad en el contexto del papel que juegan los inversionistas institucionales en el mercado accionario colombiano se toma como un mecanismo para medir la importancia sistémica de cada uno de ellos, esto es, una falla en su operar significaría una disminución en la solvencia y en el capital del resto de empresas de la red, en especial, en aquellas que tienen control accionario último sobre ellos, lo que significaría mayor riesgo de insolvencia en las empresas con las que se conectan y, además, pasan a ser instituciones de un importante impacto sistémico durante períodos de estrés financiero. 


\section{Gráfico 2. Nodos de centralidad Authority y Hub}

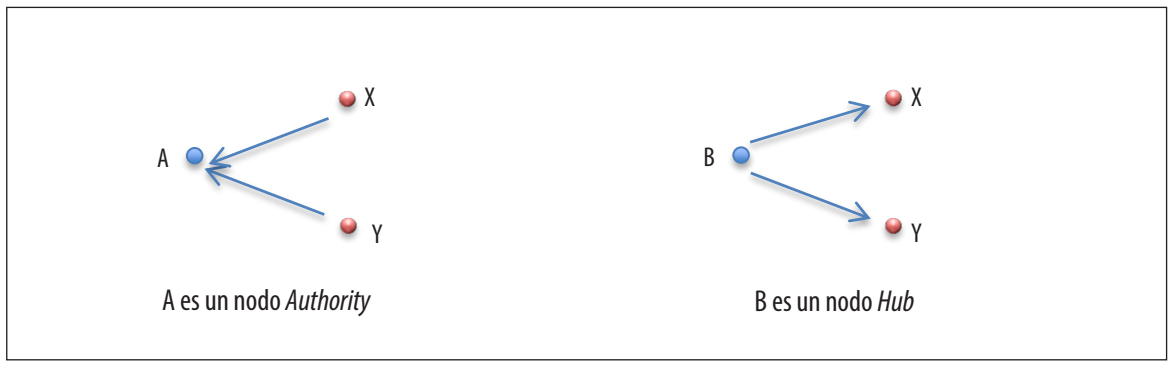

Fuente: elaboración propia

En $c_{i}$ definimos la medida de autoridad del nodo $i$, como la sumatoria ponderada de las medidas hubs, $h_{k}$, de los $k$ nodos que están conectados a $i$, siendo los nodos hubs los que cuentan con el mayor número de conexiones directas con otros nodos dentro de toda la estructura de red.

$$
c_{i}=\sum_{k} a_{k, i} h_{k}
$$

\subsection{Datos}

Los datos sobre participaciones directas de las empresas colombianas en las demás empresas de la muestra fueron tomados de la base de datos Benchmark para el año $2012^{2}$. Se cuenta con la información para 108 organizaciones ${ }^{3}$ que incluyen empresas, bancos comerciales, fondos de pensiones, corporaciones financieras y sociedades administradoras de valores; también se incluyen firmas pertenecientes a los sectores industrial, minero, de construcciones, alimentos, de servicios no financieros (donde registran compañías de televisión, telefonía, servicios domiciliarios, comercio, entre otros). Una lista detallada de las empresas analizadas está en los anexos. Se buscó incluir las trescientas empresas más grandes según el tamaño de sus activos, las cuales equivalen al $62 \%$ de los activos de todas las empresas del sector real y, además, se consideraron 100 empresas del sector financiero que tienen un peso del 95\% en el total de activos de ese sector. Luego de filtrar la base, de acuerdo con las empresas que tuvieran la información requerida sobre participaciones accionaras cruzadas, la muestra final quedó reducida a 108 empresas.

2 Quienes estén interesados en la base de datos utilizada en este estudio pueden solicitarla al correo electrónico jorge.uribe@correounivalle.edu.co

3 En el anexo, tabla 6, se presenta la lista de las empresas que fueron incluidas en el estudio. 


\section{PRINCIPALES RESULTADOS}

El procesamiento de la información de la matriz de propiedad integrada permite mostrar las principales características generales de la red (ver tabla 1).

Tabla 1. Principales características de la red

\begin{tabular}{|l|c|}
\hline \multicolumn{1}{|c|}{ Característica } & Resultado \\
\hline Número de empresas & 108 \\
\hline Número de participaciones (conexiones) & 188 \\
\hline \multicolumn{1}{|c|}{ Tipo de medida } & Resultado de la medida \\
\hline Grado medio & 4 \\
\hline Diámetro de la red & 5 \\
\hline Componentes conexos (weakly) & 11 \\
\hline
\end{tabular}

Fuente: elaboración propia

Las empresas de la red presentan, en promedio, participación accionaria en cuatro empresas. El diámetro de la red indica la distancia más larga entre dos empresas, que en este caso es de 5 nodos. Se identifica que el 10\% de las empresas de la red se encuentran débilmente conectadas.

Por otro lado, del total de 108 empresas, tres son fondos de pensiones los que presentan los mayores grados nodales, siendo el grado de salida muy superior al de entrada. Lo anterior evidencia que dichos inversionistas institucionales tienen control accionario último en un número importante de otras empresa de la muestra de datos (ver gráfico 3).

Para la Administradora de Fondos de Pensiones y Cesantías Protección el grado de salida es de 22, lo que significa que esta entidad posee control accionario último en otras 22 empresas mientras que la Sociedad Administradora de Fondos de Pensiones y Cesantías Porvenir presenta propiedad integrada en 20. Otro caso, también de un inversionista institucional, es el de Colfondos S. A. Pensiones y Cesantías que cuenta con control accionario último en 20 firmas. 
Gráfico 3. Estructura de red resaltando las empresas con mayores grados nodales

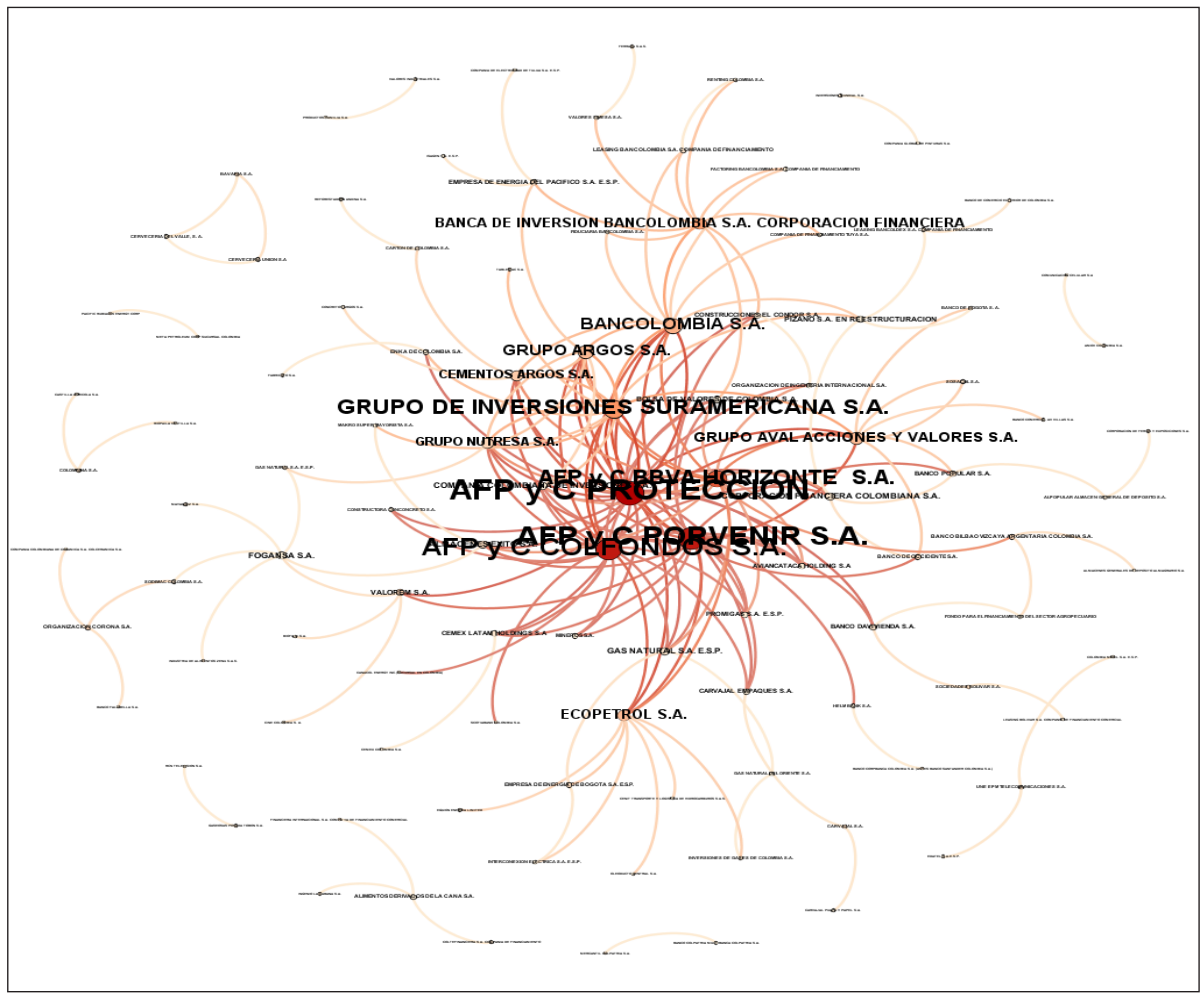

Fuente: elaboración propia en Ghepi

En términos de los grados de entrada, que determinan el número de firmas que tienen propiedad cruzada en una empresa específica, tanto para AFP Protección como para AFP Porvenir es de 2 firmas mientras que para AFP Colfondos es de 1. ¿Cuáles son las empresas que tienen control accionario último en estos fondos de pensiones? La respuesta a esta pregunta se encuentra detallada en la siguiente tabla:

Tabla 2. Principales inversionistas institucionales con mayor grado de salida

\begin{tabular}{|c|c|c|c|}
\hline Empresa & Grado de salida & & Grado de entrada \\
\hline \multirow{2}{*}{ AFP Protección } & \multirow{2}{*}{22} & \multirow{2}{*}{2} & Grupo Inversiones Suramericana S. A. \\
\hline & & & Bancolombia S. A. \\
\hline \multirow{2}{*}{ AFP Porvenir } & \multirow{2}{*}{20} & \multirow{2}{*}{2} & Grupo AVAL A y V S. A. \\
\hline & & & Banco de Occidente S. A. \\
\hline AFP Colfondos & 20 & 1 & Scotiabank Colombia S. A. \\
\hline
\end{tabular}

Fuente: cálculos propios 
El ordenamiento ascendente de los grados de entrada para las 108 firmas muestra que las que tienen máximo valor en este indicador también son inversionistas institucionales, a saber, son el Grupo de Inversiones Suramericana S. A. y Bancolombia S. A, cada una con grado de entrada igual a 7. Como se puede observar en la red de ego del Grupo I Suramericana S. A., es la empresa en la que recae el control accionario último de cuatro fondos de pensiones, del Grupo Argos, de Cementos Argos y del Grupo Nutresa. Por su parte, en Bancolombia tienen propiedad integrada los mismos fondos de pensiones y las filiales de Argos que aparecen en el caso anterior, con la particularidad de que el Grupo I Suramericana también tiene control accionario último en Bancolombia S. A. (ver gráfico 4). Este resultado muestra que existe una fuerte relación entre inversionistas institucionales, por lo que el fallo en la actividad de alguno de ellos supone un grave riesgo para el mercado accionario colombiano, como un todo.

Gráfico 4. Redes de ego: Grupo de Inversiones Suramericana S. A. y Bancolombia S. A.
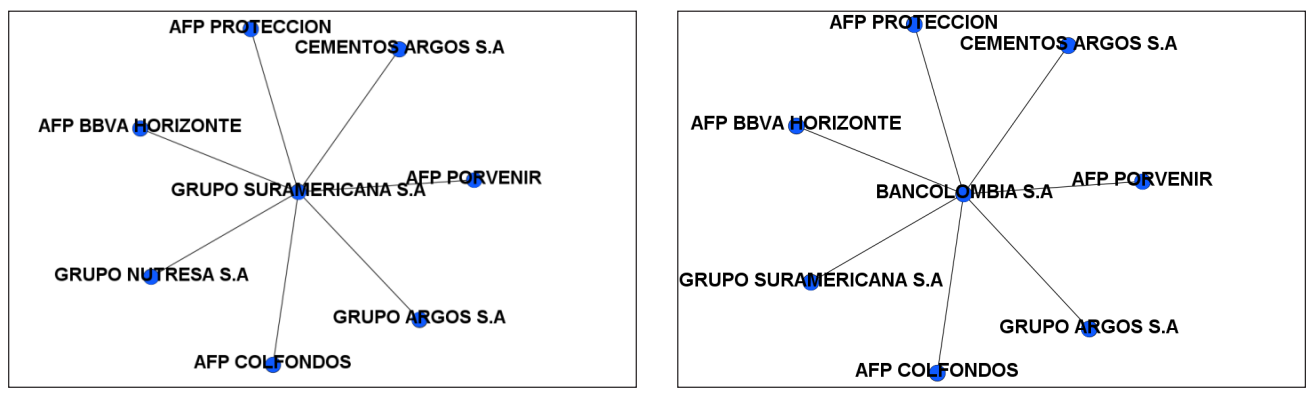

Fuente: elaboración propia en Gephi

Para el caso del indicador de autoridad, los resultados revelan que aquellas empresas con altos grados de entrada también son autoridades como es el caso del Grupo I Suramericana y el Grupo Nutresa S. A. Esto evidencia que las firmas que tienen control accionario último en ellas son firmas con mayor importancia en la estructura de red.

Entendiendo la medida de autoridad como un indicador de la importancia sistémica de cada firma, se tiene que un fallo en la operación de las firmas que son autoridades significaría una disminución en la solvencia y en el capital de las empresas que, en especial, tienen control accionario último en ellas. En la tabla que se presenta a continuación, se muestran las empresas con los cinco mayores valores de autoridad: 
Tabla 3. Empresas con alta importancia sistémica (autoridades)

\begin{tabular}{|c|c|c|c|}
\hline Autoridad & Empresa & Autoridad & Empresa \\
\hline \multirow{2}{*}{0,029} & Grupo Suramericana S. A. & \multirow{5}{*}{0,018} & Ecopetrol S. A. \\
\hline & Bancolombia S. A. & & Almacenes Éxito S. A. \\
\hline \multirow{4}{*}{0,026} & Grupo Argos S. A. & & Aviancataca Holding S. A. \\
\hline & Grupo Nutresa S. A. & & Promigas S. A. ESP \\
\hline & Fogansa S. A. & & Carvajal Empaques S. A. \\
\hline & CCIS. A. & \multirow{7}{*}{0,015} & Cemex Latam Holdings S. A. \\
\hline \multirow{6}{*}{0,022} & Grupo AVAL AyV S. A. & & Cons. El Cóndor S. A. \\
\hline & Cementos Argos S. A. & & Banco Davivienda S. A. \\
\hline & Gas Natural S. A. ESP & & OII S. A. \\
\hline & CFC S. A. & & ADC S. A. \\
\hline & BVC S. A. & & Enka de Colombia S. A. \\
\hline & Pizano S. A. & & Mineros S. A. \\
\hline
\end{tabular}

Fuente: cálculos propios

¿Qué pasaría si por choques exógenos a la estructura de red aquí analizada, los fondos de pensiones, en su papel de inversionistas institucionales, desaparecieran del mercado accionario colombiano? Los ejercicios de simular la salida de los fondos de pensiones muestran que ante la ausencia de AFP Protección, en la red total se perderían 24 enlaces, es decir, se pierde la participación integrada de 24 empresas, la red pierde densidad (menos conexiones) y se fracciona en más comunidades; algo similar sucede cuando se deja por fuera a AFP Colfondos. En el caso extremo, en el que faltaran los 4 AFP analizados, la red total perdería el control accionario último de 83 empresas y se fraccionaría en 25 comunidades (ver tabla 5).

Tabla 5. Estructura de red sin fondos de pensiones

\begin{tabular}{|l|c|c|c|c|}
\hline \multicolumn{1}{|c|}{ Caso } & Nodos & Enlaces & Densidad & Comunidades \\
\hline Red Total & 108 & 188 & 0,016 & 17 \\
\hline Se excluye AFP Protección & 107 & 164 & 0,015 & 19 \\
\hline Se excluye AFP Porvernir S.A & 107 & 166 & 0,017 & 17 \\
\hline Se excluye AFP Colfondos S.A & 107 & 167 & 0,015 & 19 \\
\hline Se excluye AFP BBVA Horizonte S. A. & 107 & 172 & 0,015 & 17 \\
\hline Se excluyen 4 AFP & 104 & 105 & 0,01 & 25 \\
\hline
\end{tabular}

Fuente: elaboración propia 


\section{CONCLUSIONES}

En este documento se motiva el estudio de las características sistémicas de los inversionistas institucionales colombianos, en particular los fondos de pensiones y, en menor medida, los bancos comerciales que, si bien por legislación no pueden invertir en acciones, sí representan una buena parte de la oferta de títulos participativos en el mercado. Se explora, mediante la construcción de matrices de control de propiedad y redes sociales, la centralidad de estas instituciones en el mercado de acciones colombiano.

Este análisis permite identificar las entidades con mayores nodos de entrada y de salida. Las primeras son instituciones que en caso de quiebra podrían afectar a un gran número de entidades dentro de la red, mientras que las segundas son entidades que reciben múltiples choques de distintas entidades en la red. Este análisis, junto con ejercicios de simulación, permite resaltar la importancia de los fondos de pensiones como agentes de importancia sistémica para el mercado de acciones colombiano. Es a través de estos fondos como la red se construye, puesto que tienen participaciones importantes en el resto de empresas de la muestra. De la misma forma, debido a las participaciones accionarias cruzadas, una eventual falla de alguna de estas entidades implicaría un choque para el mercado de elevada magnitud, que haría difícil preservar la estabilidad del mismo.

Estos resultados motivan un avance pertinente en la legislación, por ejemplo, sobre la determinación de niveles adecuados de solvencia para estas entidades, que tenga en cuenta su papel como instituciones financieras sistémicas.

Por supuesto, este es tan solo un paso en la caracterización adecuada de las estructuras que conforman el mercado de acciones colombiano y no agota el tema de estudio, que ya ha sido liderado por el trabajo de Gutiérrez, Pombo y Taborda (2008). Cualquier ampliación de la base de datos, más allá de los registros públicos de las empresas, sobre su control cruzado accionario en otras instituciones, enriquecerá el análisis de la estructura de red. También es posible estudiar las matrices de propiedad directa, de forma dinámica, para observar la evolución específica en las formas de propagación de los choques dentro del sistema.

\section{BIBLIOGRAFÍA}

Acharya, Viral; Pedersen, Lasse; Philippon, Thomas y Richardson, Matthew (2010). Measuring systemic risk. En: Working paper, New York University, 46p.

Adrian, Tobias y Brunnermeier, Markus (2014). CoVar. En: Federal Reserve Bank of New York, Staff Reports, No. 348, 53p. 
Relevancia de los inversionistas institucionales en el mercado accionario colombiano

Basak, Suleyman y Pavlova, Anna (2013). Asset prices and institutional investors. En: American Economic Review, Vol. 103, No. 5, p. 1728-1758.

Bohl, Martin; Brzeszczynski, Janusz y Wilfling, Bern (2009). Institutional investors and stock returns volatility: Empirical evidence from a natural experiment. En: Journal of Financial Stability, Vol. 5, No. 2, p. 170-182.

Brunnermeier, Markus y Nagel, Stefan (2004). Hedge funds and the technology bubble. En: The Journal of Finances, Vol. 59, No. 5, p. 2013-2040.

Cabrera, Wilmar; Melo, Luis y Parra, Daniel (2014). Relación entre el riesgo sistémico del sistema financiero y el sector real: un enfoque FAVAR. En: Revista Ensayos Sobre Política Económica (ESPE), Vol. 32, No. 75, p. 1-22.

Cabrera, Wilmar; Corredor, Adriana y Quicazan, Carlos (2012). Requerimientos macroprudenciales de capital y riesgo sistémico: una aplicación para Colombia. En: Reportes de Estabilidad Financiera, Banco de la República, No, 74, 18p.

Campbell, Johan; Ramadorai, Tarun y Schwartz, Allie (2009). Caught on tape: Institutional trading, stock returns, and earnings announcements. En: Journal of Financial Economics, Vol. 92, No. 1, p. 66-91.

Cepeda, Freddy (2008). La topología de redes como herramienta de seguimiento en el sistema de pagos de alto valor en Colombia. En: Borradores de Economía, Banco de la República, No. 513, 31p.

Chan-Lau, Jorge (2005). Pension funds and emerging markets. En: Financial Markets, Institutions E Instruments, Vol. 14, No. 3, p. 107-134.

Chen, Honghui; Noronha, Gregory y Singal, Vijay (2004). The price response to SEP 500 index additions and deletions: Evidence of asymmetry and a new explanation. En: The Journal of Finance, Vol. 59, No. 4, p. 1901-1930.

Choe, Hyuk; Kho, Bong-Chan y Stulz, Ren (1999). Do foreign investors destabilize stock markets? The Korean experience in 1997. En: Journal of Financial Economics, Vol. 54, No 2, p. 227-264.

Cohen, Randolph; Gompers, Paul y Vuolteenaho, Tuomo (2002). Who underreacts to cash-flow news? Evidence from trading between individuals and institutions. En: Journal of Financial Economics, Vol. 66, No 2-3, p. 409-462.

Davis, E. Philip y Hu, Yu-Wei (2005). Is There A Link Between Pension-Fund Assets And Economic Growth? A Cross-Country Study. En: Brunel University and NIESR, No. 04-23, 32p.

Dennis, Patrick y Strickland, Deon (2002). Who Blinks in Volatile Markets, Individuals or Institutions? En: The Journal of Finance, Vol. 57, No. 5, p. 1923-1949.

Gabaix, Xavier; Gopikrishnan, Parameswaran; Plerou, Vasiliki y Stanley, H. Eugene (2006). Institutional Investors and Stock Market Volatility. En: The Quarterly Journal of Economics, Vol. 121, No. 2, p. 461-504.

Glattfelder, James (2013). Decoding Complexity: Uncovering Patterns in Economic Networks. Springer Theses, 228p. DOI: 10.1007/978-3-642-33424-5. 
Gompers, Paul y Metrick, Andrew (2001). Institutional investors and equity prices. En: The Quarterly Journal of Economics, Vol. 116, No. 1, p. 229-259.

Grossman, Sanford y Hart, Oliver (1986). The Costs and Benefits of Ownership: A Theory of Vertical and Lateral Integration. En: Journal of Political Economy, Vol. 94, No. 4, p. 691-719.

Gutiérrez, Luis; Pombo, Carlos y Taborda, Rodrigo (2008). Ownership and control in Colombian corporations. En: The Quarterly Review of Economics and Finance, Vol. 48, No 1, p. 22-47.

Harris, Lawrence y Gurel, Eitan (1986). Price and volume effects associated with changes in the SEP 500 List: New evidence for the existence of price pressures. En: The Journal of Finance, Vol. 41, No 4, p. 815-829.

Hvidkjaer, Soeren (2006). A Trade-Based analysis of momentum. En: The Review of Financial Studies, Vol. 19, No 2, p. 457-491.

Kleinberg, Jon (1999). Authoritative sources in a hyperlinked environment. En: Journal of the ACM, Vol. 46, No. 5, p. 604-632.

Lakonishok, Josef; Shleifer, Andrei y Vishny, Robert (1992). The impact of institutional trading on stock prices. En: Journal of Financial Economics, Vol. 32, No. 1, p. 23-43.

León, Carlos; Machado, Clara y Sarmiento, Miguel (2014). Identifying central bank liquidity super-spreaders in interbank funds networks. En: Borradores de Economía, Banco de la República, No. 816, 28p.

León, Carlos y Pérez, Jhonatan (2013). Assessing financial market infrastructures' systemic importance with authority and hub centrality. En: Journal of Financial Market Infrastructures, Vol. 2, No. 3, p. 67-87.

Machado, Clara; León, Carlos; Sarmiento, Miguel; Cepeda, Freddy; Chipatecua, Orlando y Cely, Jorge (2011). Riesgo sistémico y estabilidad del sistema de pagos de alto valor en Colombia: análisis bajo topología de redes y simulación de pagos. En: Ensayos sobre Política Económica, Vol. 29, No. 65, p. 107-175.

Niggemann, Taro y Rocholl, Jörg (2010). Pension funding and capital market development. En Working Paper, ESMT, 43p.

Pérez, Jhonatan; León, Carlos y Mariño, Ricardo (2015). Aproximación a la estructura del mercado cambiario colombiano desde el análisis de redes. En: Revista Ciencias Estratégicas, Vol. 22, No. 32, p. 261-278.

Schmidt-Hebbel, Klauss (1999). Does Pension Reform Really Spur Productivity, Saving, and Growth? En: Documentos de trabajo del Banco Central de Chile, No. 33, 45p.

Shleifer, Andrei (1986). Do demand curves for stocks slope down? En:The Journal of Finance, Vol. 41, No. 3, p. 579-590.

Sias, R.ichard (1996). Volatility and the Institutional Investor. En: Financial Analysts Journal, Vol. 52, No. 2, p. 13-20.

Thomas, Ashok; Spataro, Luca y Mathew, Nandhita (2014). Pension funds and stock market volatility: An empirical analysis of OECD countries. En: Journal of Financial Stability, Vol. 11, No. 1, p. 92-103. 
Relevancia de los inversionistas institucionales en el mercado accionario colombiano

Uribe, Jorge y Fernández, Julián (2014). Riesgo sistémico en el mercado de acciones Colombiano: alternativas de diversificación bajo eventos extremos. En: Cuadernos de Economía, Vol. 33, No. 63, p. 613-634.

Uribe, Jorge; Mosquera, Stefania y Restrepo, Natalia (2013). Mercado de acciones colombiano. Determinantes macroeconómicos y papel de las AFP. En: Sociedad y Economía, No. 24, p. 207-229. 


\section{ANEXO}

Tabla 6. Empresas incluidas en la muestra de estudio

\begin{tabular}{|c|c|c|c|c|c|}
\hline Id & \multicolumn{5}{|c|}{ Nombre de la empresa } \\
\hline 1 & Cemex Latam Holdings S. A. & 37 & $\begin{array}{l}\text { Fondo para el Financiamiento del } \\
\text { Sector Agropecuario }\end{array}$ & 73 & Edatel S. A. ESP \\
\hline 2 & Gas Natural S. A. ESP & 38 & Fiduciaria Bancolombia S. A. & 74 & Mineros S. A. \\
\hline 3 & $\begin{array}{l}\text { Inversiones de Gases de Colombia } \\
\text { S. A. }\end{array}$ & 39 & $\begin{array}{l}\text { Organización de Ingeniería } \\
\text { Internacional S. A. }\end{array}$ & 75 & $\begin{array}{l}\text { Companía de Electricidad de Tuluá } \\
\text { S. A. Esp }\end{array}$ \\
\hline 4 & $\begin{array}{l}\text { Administradora de Fondos de } \\
\text { Pensiones y Cesantías Protección }\end{array}$ & 40 & Oleoducto Central S. A. & 76 & $\begin{array}{l}\text { Almacenes Generales de Depósito } \\
\text { Almagrario S. A. }\end{array}$ \\
\hline 5 & $\begin{array}{l}\text { Sociedad Administradora de Fondos } \\
\text { de Pensiones y Cesantías Porvenir } \\
\text { S. A. }\end{array}$ & 41 & Isagen S. A. ESP & 77 & Banco Falabella S. A. \\
\hline 6 & $\begin{array}{l}\text { BBVA Horizonte Sociedad } \\
\text { Administradora de Fondos de } \\
\text { Pensiones y Cesantías S. A. }\end{array}$ & 42 & Renting Colombia S. A. & 78 & Makro Súper Mayorista S. A. \\
\hline 7 & Sosacol S. A. & 43 & Valores Simesa S. A. & 79 & Riopaila Castilla S. A. \\
\hline 8 & $\begin{array}{l}\text { Colfondos S. A. Pensiones y } \\
\text { Cesantías }\end{array}$ & 44 & Fogansa S. A. & 80 & Cervecería del Valle S. A. \\
\hline 9 & Grupo Aval Acciones y Valores S. A. & 45 & $\begin{array}{l}\text { Compañía Colombiana de } \\
\text { Inversiones S. A. }\end{array}$ & 81 & $\begin{array}{l}\text { Canacol Energy INC.(sucursal en } \\
\text { Colombia) }\end{array}$ \\
\hline 10 & $\begin{array}{l}\text { Leasing Bancoldex S. A. Compañía } \\
\text { de Financiamiento }\end{array}$ & 46 & $\begin{array}{l}\text { Alimentos Derivados de la Caña } \\
\text { S. A. }\end{array}$ & 82 & $\begin{array}{l}\text { Cenit Transporte y Logística de } \\
\text { Hidrocarburos S. A. S. }\end{array}$ \\
\hline 11 & $\begin{array}{l}\text { Banca de Inversión Bancolombia S. } \\
\text { A. Corporación Financiera }\end{array}$ & 47 & RCN Televisión S. A. & 83 & Comunicación Celular S. A \\
\hline 12 & Sodimac Colombia S. A. & 48 & Bolsa de Valores de Colombia S. A. & 84 & Castilla Agrícola S. A. \\
\hline 13 & $\begin{array}{l}\text { Empresa de Energía del Pacífico S. } \\
\text { A. ESP }\end{array}$ & 49 & RCN Televisión S. A. & 85 & Bavaria S. A. \\
\hline 14 & $\begin{array}{l}\text { Grupo de Inversiones Suramericana } \\
\text { S. A. }\end{array}$ & 50 & $\begin{array}{l}\text { Meta Petroleum Corp Sucursal } \\
\text { Colombia }\end{array}$ & 86 & $\begin{array}{l}\text { Banco de Comercio Exterior de } \\
\text { Colombia S. A. }\end{array}$ \\
\hline 15 & $\begin{array}{l}\text { Compañía Nacional De Chocolate } \\
\text { S. A. S. }\end{array}$ & 51 & Equion Energía Limited & 87 & Valores Industriales S. A. \\
\hline 16 & Valorem S. A. & 52 & Cemex Colombia S. A. & 88 & Industria de Alimentos Zenú S. A. S. \\
\hline 17 & Banco de Bogotá S. A. & 53 & $\begin{array}{l}\text { Compañía Colombiana de Cerámica } \\
\text { S. A. Colcerámica }\end{array}$ & 89 & Sociedades Bolivar S. A. \\
\hline 18 & Banco Popular S. A. & 54 & Pizano S. A. En reestructuración & 90 & Mercantil Colpatria S. A. \\
\hline 19 & Banco Davivienda S. A. & 55 & Helm Bank S. A. & 91 & $\begin{array}{l}\text { Corporación de Ferias y } \\
\text { Exposiciones S. A. }\end{array}$ \\
\hline
\end{tabular}


Relevancia de los inversionistas institucionales en el mercado accionario colombiano

\begin{tabular}{|c|c|c|c|c|c|}
\hline Id & \multicolumn{5}{|c|}{ Nombre de la empresa } \\
\hline 20 & $\begin{array}{l}\text { Leasing Bancolombia S. A. } \\
\text { Compañía de Financiamiento }\end{array}$ & 56 & Interconexión Eléctrica S. A. ESP & 92 & Organización Corona S. A. \\
\hline 21 & Cementos Argos S. A. & 57 & $\begin{array}{l}\text { Alpopular Almacén General de } \\
\text { Depósito S. A. }\end{array}$ & 93 & $\begin{array}{l}\text { Banco Bilbao Vizcaya Argentaria } \\
\text { Colombia S. A. }\end{array}$ \\
\hline 22 & Banco de Occidente S. A. & 58 & $\begin{array}{l}\text { Compañía de Financiamiento Tuya } \\
\text { S. A. }\end{array}$ & 94 & Inversiones Mundial S. A. \\
\hline 23 & Cartón de Colombia S. A. & 59 & $\begin{array}{l}\text { Banco Colpatria Multibanca } \\
\text { Colpatria S. A. }\end{array}$ & 95 & Scotiabank Colombia S. A. \\
\hline 24 & $\begin{array}{l}\text { Corporación Financiera Colombiana } \\
\text { S. A. }\end{array}$ & 60 & Banco Comercial AV Villas S. A. & 96 & $\begin{array}{l}\text { Financiera Internacional S. A. } \\
\text { Compañía de Financiamiento } \\
\text { Comercial }\end{array}$ \\
\hline 25 & Grupo Nutresa S. A. & 61 & $\begin{array}{l}\text { Leasing Bolivar S. A. Compañía de } \\
\text { Financiamiento Comercial }\end{array}$ & 97 & Carvajal S. A. \\
\hline 26 & Cervecería Unión S. A & 62 & Concretos Argos S. A. & 98 & Mayaguez S. A. \\
\hline 27 & Grupo Argos S. A. & 63 & Promigas S. A. ESP & 99 & Fabricato S. A. \\
\hline 28 & Almacenes Éxito S. A. & 64 & Gas Natural del Oriente S. A. & 100 & $\begin{array}{l}\text { Banco Corpbanca Colombia S. A. } \\
\text { (antes Banco Santander Colombia } \\
\text { S. A.) }\end{array}$ \\
\hline 29 & Bancolombia S. A. & 65 & Carvajal Pulpa y Papel S. A. & 101 & Gaseosas Posada Tobón S. A. \\
\hline 30 & Construcciones El Cóndor S. A. & 66 & Reforestadora Andina S. A. & 102 & $\begin{array}{l}\text { Coltefinanciera S. A. Compañía de } \\
\text { Financiamiento }\end{array}$ \\
\hline 31 & $\begin{array}{l}\text { Factoring Bancolombia S. A. } \\
\text { Compañía de Financiamiento }\end{array}$ & 67 & Carvajal Empaques S. A. & 103 & Ferrasa S. A. S. \\
\hline 32 & Ecopetrol S. A. & 68 & Cine Colombia S. A. & 104 & Ingenio la Cabaña S. A. \\
\hline 33 & $\begin{array}{l}\text { Empresa de Energía de Bogotá S. } \\
\text { A. ESP }\end{array}$ & 69 & Compañía Global de Pinturas S. A. & 105 & Amov Colombia S. A. \\
\hline 34 & Aviancataca Holding S. A. & 70 & Productos Familia S. A. & 106 & UNE EPM Telecomunicaciones S. A \\
\hline 35 & Tablemac S. A. & 71 & Constructora Conconcreto S. A. & 107 & Pacific Rubiales Energy Corp. \\
\hline 36 & Biofilm S. A. & 72 & Enka de Colombia S. A. & 108 & Colombina S. A. \\
\hline
\end{tabular}

Fuente: elaboración propia 